\title{
Torsional buckling of thin-walled cylinders under circumferentially varying thermal loads
}

\author{
Kara M. Charles* and Anthony M. Waas ${ }^{\dagger}$ \\ University of Michigan, Ann Arbor, Michigan, 48109-2140
}

\begin{abstract}
Thin-walled cylinders, used in a variety of engineering applications, are often subjected to situations where the applied loading is not limited to a single loading type. The introduction of multiple loads alters the stability characteristics of the system in a manner that must be understood to maintain structural integrity and abide by safety regulations. The present study investigates the elastic buckling response of thin-walled cylindrical shells under a combination of torsional loads and circumferentially-varying thermal loads.
\end{abstract}

\section{Nomenclature}

$E \quad=$ Young's modulus

$v \quad=$ Poisson's ratio

$\alpha=$ coefficient of thermal expansion

$L \quad=$ axial length of cylinder

$a \quad=$ radius of cylinder

$h \quad=$ thickness of shell wall

$m \quad=$ number of axial half sine waves

$n=$ number of circumferential full sine waves

$N_{X}, N_{\theta} \quad=$ in-plane direct stress resultants

$N_{X \theta} \quad=$ in-plane shear stress resultant

$M \quad=$ in-plane moment resultants

$\tau=$ torsional load

$w \quad=$ out of plane displacement

$x=$ axial cylindrical coordinate

$\theta=$ circumferential cylindrical coordinate

$r \quad=$ radial cylindrical coordinate

$T_{o} \quad=$ mean thermal load

$\delta \quad=$ maximum temperature difference

()$_{, q} \quad=$ first derivative of quantity with respect to variable $\mathrm{q}$

\section{Introduction}

$\mathrm{T}$ Thin-walled cylinders are used in a variety of engineering applications, such as airplane fuselages, submarines, building construction, and manufacturing machinery. Often the applications are in situations where the loading cannot be limited to a single loading type such as axial compression, torsion, or pressure. ${ }^{1}$ The introduction of multiple loading alters the stability characteristics of the system in a manner which must be understood to maintain structural integrity and abide by safety regulations.

The buckling behavior of thin-walled cylindrical shells is understood for simple torsional loading, where classical elastic buckling theory uses the potential energy approach to describe the stability of the shell. ${ }^{2}$ Donnell equations, described in Refs. 3 and 4, are frequently used to determine the deformation of a cylindrical shell under combined loads. This study investigates the influence of thermal loads on the buckling behavior of thin-walled cylindrical shells subjected to torsional loads.

\footnotetext{
* Research Assistant, Department of Aerospace Engineering, 1320 Beal St. Ann Arbor, MI 48109, AIAA Student Member

${ }^{\dagger}$ Professor, Department of Aerospace Engineering, 1320 Beal St. Ann Arbor, MI 48109, Associate Fellow, AIAA 
The motivation for the present investigation, including thermal forces, stems from an interest in examining the behavior of paper mill drying cylinders. ${ }^{6,7}$ These cast-iron, steam-heated pressure vessels are typically 150 to 300 inches in length and have a radius of 6 to 9 feet. Since their shell wall thickness is on the order of 2-3 inches, these dryers can be analyzed as thin-walled cylinders. In paper machines, the dryers are positioned with their center axis of rotation horizontal with the floor. The dryer cylinder rotates at a frequency of up to 2 revolutions/second as moist paper is pressed on the surface to be dried via evaporation. In most dryers, an evenly spaced condensate removal system inside the structure extracts condensate from the internal surface. Typically, dryers have four or six locations where condensate is removed from the dryer. The action of the condensate removal and excess steam passing through the system creates bands of cooler and warmer temperatures in the shell, running axially along the length of the cylinder. A torsional load is introduced into the paper drying system by a linear load from a "crepe blade" which scrapes the dried paper from the cylinder. Since the rotation is driven from only one side of the cylinder, the tangential portion of the linear load resists the rotation of the system, resulting in a torsional load in the cylinder.

On occasion, paper dryers have experienced a sudden and dangerous instability. When the instability occurs, the dryer shell distortion increases by an order of magnitude, compromising the operation of the equipment and the safety of the operators. It is the common belief in the industry that this is due to the thermal variation around the dryer. Whereas the temperature gradient does create deflection of the cylinder surface, dryers with both four- and six-node thermal variations are found to buckle into a five-node distortion pattern, indicating that the thermal loading is not the primary driving factor in the structural instability. One explanation is that the thermal distortion resulting from the normal action of the condensate removal system increases the torsional load produced by the crepe blade, ultimately reaching the critical load and producing torsional buckling of the dryer shell.

In the present study, the thermal load investigated is applied as a sinusoidal temperature variation about the circumference, where the median temperature, amplitude of oscillation and the number of cycles around the cylinder are maintained as variables. The individual loading conditions are first considered separately then together in the combined loading state. The system is examined in such a way that the thermal loads are assumed to influence the behavior of the critical torsional buckling load.

The present study is carried out under conditions of plane stress, according to the thin-walled shell assumption. The influence of thermal loading on the pre-bucked state of the cylinder is examined. Total strains are found by adding the thermal strain component to the mechanical strain using linear superposition. The second derivatives of the kinematic relations are manipulated to yield a relation between the strains and surface deflection. An Airy stress function is introduced and utilized to determine the resultant loads for the cylinder with circumferentially varying thermal loading. With this considered, the combined thermal and torsional loading buckling problem is solved by using a Galerkin approximation. The results from the analyses show the influence of thermal loads on the torsional buckling characteristics of thin walled cylinders. When the solutions are specialized to the case of the steam heated pressure vessels, good agreement is found between the analysis and the observed buckling modes.

\section{Fundamental equations for the thin-walled cylinder}

The analysis that is described in the present study considers thin-walled cylinders of isotropic materials. Directional properties relating to ply direction of a composite laminate can be integrated into the equations for an analysis of cylinders of orthotropic materials. However, for simplicity, the isotropic material assumption has been made.

The following figure describes the shell in question, where $h$ is the thickness, $L$ is the length, and $a$ is the undeformed-middle-surface radius. For this shell to be considered thin-walled, $h$ must be considerably less than $a$.

The thin-walled assumption allows the analysis to be carried out under conditions of plane stress. Whereas this is a good approximation of the true conditions, it also simplifies the equations and the solution significantly.

The study of the behavior of a thin-walled cylinder under both thermal and torsional loading begins by examining force and moment equilibrium of a shell wall element, which provides Eqs. (1).

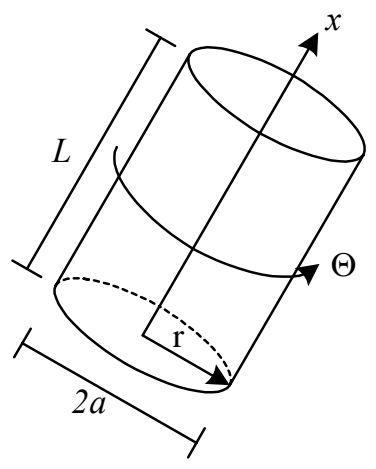

Figure 1: Circular cylindrical shell coordinate system reference diagram 


$$
\begin{gathered}
a N_{x, x}+N_{x \theta, \theta}=0 \\
a N_{x \theta, x}+N_{\theta, \theta}=0 \\
a^{2} M_{x, x x}+2 a M_{x \theta, x \theta}+M_{\theta, \theta \theta}-a N_{\theta}-a^{2} N_{x} \beta_{x, x}-a N_{x \theta}\left(a \beta_{\theta, x}+\beta_{x, \theta}\right)=-p a^{2}
\end{gathered}
$$

where $\mathrm{p}$ is the radial pressure, defined positive outward, and the forces and moments are described by the following constitutive Eqs. (2) involving the in-plane force and moment resultants (integrated over the thickness of the shell), mid-plane strains and curvatures of the shell.

$$
\begin{aligned}
& N_{x}=C\left(\varepsilon_{x}+v \varepsilon_{\theta}\right) \\
& N_{\theta}=C\left(\varepsilon_{\theta}+v \varepsilon_{x}\right) \\
& N_{x \theta}=C \frac{1-v}{2} \gamma_{x \theta} \\
& M_{x}=D\left(\kappa_{x}+v \kappa_{\theta}\right) \\
& M_{\theta}=D\left(\kappa_{\theta}+v \kappa_{x}\right) \\
& M_{x \theta}=D(1-v) \kappa_{x \theta}
\end{aligned}
$$

Equations (2) utilize the common structural rigidity constants $C=E h /\left(1-v^{2}\right)$ and $D=E h^{3} /\left[12\left(1-v^{2}\right)\right]$. The formulas for the strains and curvatures of the circular cylindrical shell are defined by the following Donnell kinematic relations, Eqs. (3).

$$
\begin{gathered}
\varepsilon_{x}=u_{, x}+\frac{1}{2} \beta_{x}{ }^{2} \\
\varepsilon_{\theta}=\frac{v_{, \theta}+w}{a}+\frac{1}{2} \beta_{\theta}{ }^{2} \\
\gamma_{x \theta}=\frac{u_{, \theta}}{a}+v_{, x}+\beta_{x} \beta_{\theta} \\
\kappa_{x}=\beta_{x, x} \\
\kappa_{\theta}=\frac{\beta_{\theta, \theta}}{a} \\
\kappa_{x \theta}=\frac{1}{2}\left(\frac{\beta_{x, \theta}}{a}+\beta_{\theta, x}\right)
\end{gathered}
$$

where the $\beta$ 's are angles of rotation of the normal to the shell mid-surface and are assumed to be small.

$$
\begin{gathered}
\beta_{x}=-w_{, x} \\
\beta_{\theta}=\frac{-w_{, \theta}}{a}
\end{gathered}
$$

\section{Effect of thermal loading}

The thermal loading considered in the present study is applied as a sinusoidal temperature variation around the circumference of the cylinder, such as is introduced in the paper dryer shell by the condensate removal system. The median temperature, amplitude of variation and the number of cycles around the cylinder are maintained as variables to facilitate modeling multiple thermal loading scenarios. The application of the thermal load profile is relevant to many other situations involving structures. For instance, aircraft fuselages undergo a wide range of solar thermal loading while withstanding torsional loads from wind and control surfaces. Also, the radiation to which space 
satellite structures are exposed can lead to large temperature variations through the system that, when combined with forces from propulsion, may lead to structural instabilities.

The total strain on the surface when a thermal load is introduced will be the sum of the mechanical strain and the thermal strain in both the $x$ and $\theta$ directions. The shear strain will not be effected by the thermal loading and will remain solely the mechanical strain in the $x-\theta$ direction.

The mechanical strain is determined by evaluating the simultaneous equations for the normal load from Eqs. (2) and solving for the strain components.

$$
\begin{gathered}
\varepsilon_{x, \text { mech }}=\frac{N_{x}}{E h}-\frac{v N_{\theta}}{E h} \\
\varepsilon_{\theta, \text { mech }}=\frac{N_{\theta}}{E h}-\frac{v N_{x}}{E h} \\
\gamma_{x \theta \text { mech }}=\frac{2 N_{x \theta}}{E h}
\end{gathered}
$$

The total strains can then be found by adding the thermal strain component, $\alpha \Delta T(\theta)$, to the mechanical strains in the axial and circumferential directions.

$$
\begin{gathered}
\varepsilon_{x}=\frac{N_{x}}{E h}-\frac{v N_{\theta}}{E h}+\alpha \Delta T(\theta) \\
\varepsilon_{\theta}=\frac{N_{\theta}}{E h}-\frac{v N_{x}}{E h}+\alpha \Delta T(\theta) \\
\gamma_{x \theta}=\frac{2 N_{x \theta}}{E h}
\end{gathered}
$$

The second derivatives of the kinematic relations, Eqs. (3), are found to be

$$
\begin{gathered}
\frac{\partial^{2} \varepsilon_{x}}{\partial \theta^{2}}=\frac{\partial^{3} u}{\partial x \partial \theta^{2}} \\
\frac{\partial^{2} \varepsilon_{\theta}}{\partial x^{2}}=\frac{1}{a} \frac{\partial^{3} v}{\partial x^{2} \partial \theta}+\frac{1}{a} w_{, x x} \\
\frac{\partial^{2} \gamma_{x \theta}}{\partial x \partial \theta}=\frac{1}{a} \frac{\partial^{3} u}{\partial x \partial \theta^{2}}+\frac{\partial^{3} v}{\partial x^{2} \partial \theta}
\end{gathered}
$$

which can be manipulated by substitution of the first two equations into the third equation to yield a relation between the strains and the mid-surface deflection.

$$
\frac{\partial^{2} \gamma_{x \theta}}{\partial x \partial \theta}=\frac{1}{a} \frac{\partial^{2} \varepsilon_{x}}{\partial \theta^{2}}+a \frac{\partial^{2} \varepsilon_{\theta}}{\partial x^{2}}-w_{, x x}
$$

To simplify the solution, an Airy stress function, $f(x, \theta)$, is introduced, which is defined to satisfy Eqs. (1) identically. The function relates to the in-plane stress resultants such that

$$
\begin{gathered}
N_{x}=f_{, \theta \theta} \\
N_{\theta}=a^{2} f_{, x x} \\
N_{x \theta}=-a f_{, x \theta}
\end{gathered}
$$


After the normal loads in the strain equations are replaced by the relations in Eqs. (6) and Eqs. (9), the straindeflection relation, Eq. (8), is determined.

$$
w_{, x x}=\frac{a^{2}}{E h} f_{, x x x x}+\frac{2 a}{E h}(1-2 v) f_{, x x \theta \theta}+\frac{1}{a E h} f_{, \theta \theta \theta \theta}+\frac{\alpha}{a} \Delta T_{, \theta \theta}+a \alpha \Delta T_{, x x}
$$

This study is specific to thermal loading which varies only around the circumference. That is, $\Delta T(x, \theta)=\Delta T(\theta)$. The temperature distribution of interest can vary sinusoidally around the cylinder as follows:

$$
\Delta T(\theta)=T_{o}+\frac{\delta}{2} \sin k \theta
$$

where the integer $k$ represents the number of full sine waves in the thermal loading around the circumference, and $\delta$ represents the amplitude of the thermal variation. It can be assumed that the stress function will take the form of the temperature loading that is applied to the shell.

$$
f(\theta)=f_{o}+f_{1} \sin k \theta
$$

Substituting these formulas into the strain-deflection relation, Eq. (10), leads to the simplification

$$
\frac{f_{1} k^{4}}{a E h} \sin k \theta-\frac{\delta \alpha k^{2}}{2 a} \sin k \theta=0
$$

Solving for the amplitude constant in the stress function, $f_{l}$, leads to

$$
f(\theta)=f_{o}+\frac{\delta \alpha E h}{2 k^{2}} \sin k \theta
$$

This function can then be used to determine the resultant loads for the cylinder with circumferentially-varying thermal loading. To complete the model, a uniform axial loading must be included, to account for the compressive reaction force at the boundaries of the shell. The cylinder is assumed to be sufficiently long such that boundary effects may be neglected and this uniform loading will not appear in either the circumferential or shear force terms.

$$
\begin{gathered}
N_{x}=-N_{x o}-\frac{\delta \alpha E h}{2} \sin k \theta \\
N_{\theta}=0 \\
N_{x \theta}=0
\end{gathered}
$$

\section{The torsional buckling problem}

With the thermal loading fully defined, the next step is to apply this information to the torsional buckling problem. The Donnell stability equation, Eq. (16), which is described in terms of the lateral displacement, will be used to solve for the critical buckling load.

$$
D \nabla^{8} w+\frac{E h}{a^{2}} w_{, x x x x}-\nabla^{4}\left(N_{x} w_{, x x}+\frac{2}{a} N_{x \theta} w_{, x \theta}+\frac{1}{a^{2}} N_{\theta} w_{, \theta \theta}\right)=0
$$


The axial normal force is that determined from the thermal loading, Eqs. (15). Also incorporated is a mechanical torsion loading, $\tau$, such that $N_{x \theta}=\tau h$. The solution to this equation can be obtained by applying the Galerkin method. In the case of a cylindrical shell, the equations needed are obtained as follows:

$$
\int_{0}^{2 \pi} \int_{0}^{L} L(w) \varphi_{m} d x d \theta=0
$$

where $L(w)$ is, in this case, the Donnell stability operator and $w$ is an assumed solution for the displacement of the buckled configuration. To accommodate the waves in the deflection that are coupled with a twisting behavior, an appropriate solution would be of the form

$$
w=k_{n} \sin (\bar{m} x-n \theta)
$$

where $\bar{m}=m \pi / L$, with $m$ being the integer defining the number of half sine waves along the length of the cylinder and $n$ defining the number of full sine waves around the circumference.

This leads to an expression for the critical torsional buckling load. This approach may be verified by comparing the solution for the case where there is only a uniform torsional load with the accepted result from Ref. [2].

$$
N_{x \theta o}=\frac{\left(a^{2} \bar{m}^{2}+4\right)^{2}}{4 \bar{m}} \frac{D}{a^{3}}+\frac{a^{3} \bar{m}^{3} E h}{4\left(a^{2} \bar{m}^{2}+4\right)^{2}}
$$

Here the circumferential wave number, $n$, is set to 2 to capture the lowest buckling load. If, however, the term is left in variable form, the solution for the torsional load becomes:

$$
N_{x \theta o}=\frac{\left(a^{2} \bar{m}+n^{2}\right)^{2}}{2 n \bar{m}} \frac{D}{a^{3}}+\frac{a^{3} \bar{m}^{3} E h}{2 n\left(a^{2} \bar{m}^{2}+n^{2}\right)^{2}}
$$

\section{Discussion of results}

The Galerkin approximation described in the previous section was incorporated into a computer code using Mathematica. With geometric and material data entered, the code solves for the critical torsional loads for a matrix of mode shapes, such that $m$ and $n$ are allowed to vary as integers. From this information, the lowest torsional buckling load can be identified as the critical torsional load for the critical mode shape.

The torsional shape function given in Eq. (18) was investigated for suitability in a combined torsional and axial loading state. The concern was that the torsional shape function would not allow consideration for the effect of axial loads on the torsional buckling. However, the analysis was performed for axial loading only, using both the torsional shape function and the classical axial shape function, Eq. (21).

$$
w=k_{n} \sin (\bar{m} x) \sin (n \theta)
$$

The calculated axial buckling load was the same for both shape functions, indicating the sufficiency of the torsional shape function for the combined loading case. So as to adhere to boundary conditions, the shape function used in the Galerkin approximation was altered from the form given by Eq. (18). The following equation is consistent with the boundary conditions which require the ends to be pinned into the original circular shape.

$$
w=k_{n} \sin (\bar{m} x-n \theta)-\frac{x}{L} \sin (\bar{m} L-n \theta)-\left(1-\frac{x}{L}\right) \sin (-n \theta)
$$


Table 1 depicts a section of the results output by the Mathematica code for a cylinder with a length of 250 inches, a radius of 108 inches, and a shell thickness of 2 inches. The material properties are those for aluminum and the thermal distribution is that of a six-noded sinusoidal wave whose average temperature of $400^{\circ} \mathrm{F}$ varies by $100^{\circ} \mathrm{F}$. The case corresponding to three circumferential wave (independent of the number of axial half-sine waves) leads to a zero denominator and is therefore labeled "Indeterminant." This occurred only for circumferential mode values equal to half of the thermal nodal shape number.

It can be seen that the lowest torsional load listed is $16.25 \cdot 10^{3} \mathrm{lb}$-in for the mode shape with seven full sinusoidal waves in the circumferential direction and two half sinusoidal waves in the axial direction. It should be noted that the wave number corresponding with the temperature distribution does not, in general, correspond to that of the buckled mode shape. It is noted that the number of circumferential waves corresponding to the buckling instability as predicted here correlates with field measurements as reported in References 6 and 7.

Table 1: Torsional buckling load $\left(10^{3} \mathrm{lb}\right.$-in) for cylinder under varying thermal load

\begin{tabular}{|c|c|c|c|c|}
\hline & & Axial Mode - H & alf Sine Wave & \\
\hline & 1 & 2 & 3 & 4 \\
\hline 1 & 1680 & 1411 & 1068 & 832 \\
\hline 2 & 263.2 & 378.9 & 387.9 & 343.4 \\
\hline 3 & Indeterminant & Indeterminant & Indeterminant & Indeterminant \\
\hline 4 & 21.66 & 43.83 & 76.39 & 97.76 \\
\hline 5 & 13.74 & 19.76 & 37.95 & 51.45 \\
\hline 气ै & 16.75 & 12.83 & 21.28 & 31.02 \\
\hline 7 & 25.45 & 12.65 & 14.5 & 21.07 \\
\hline 8 & 38.6 & 15.95 & 12.62 & 16.98 \\
\hline 9 & 56.12 & 21.64 & 13.5 & 16.43 \\
\hline 10 & 78.27 & 29.38 & 16.18 & 18.26 \\
\hline
\end{tabular}

The question that naturally follows is to what degree the thermal loading influences the torsional buckling of the cylinder. The conditions above were repeated with the thermal loading set to zero. Whereas the buckling mode is not altered by the removal of thermal loading, the critical torsional load is increased by $2.3 \cdot 10^{3} \mathrm{lb}$-in. This result is explained by appealing to the pre-buckling axial loading introduced by the thermal load, which contributes to the total strain energy of the system.

Table 2: Torsional buckling load $\left(10^{3} \mathrm{lb}\right.$-in) for cylinder under no thermal load

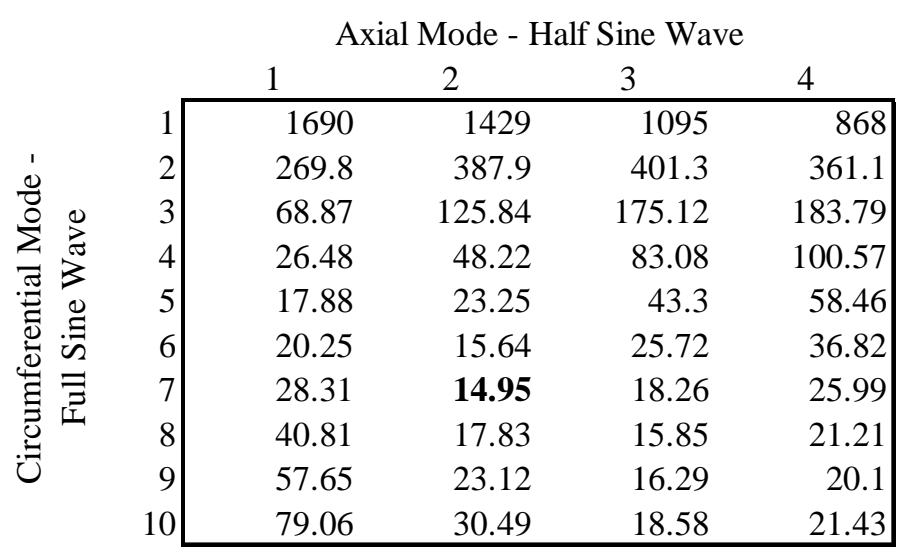

It is clear that, although the buckling mode remains unchanged in this instance, the differences between adjacent critical loads is altered. This indicates that the mode shape will change for a sufficiently high thermal loading. For the cylinder in question, the mode shape drops to six circumferential waves and two half axial waves when the 
constant thermal load nears $275^{\circ} \mathrm{F}$. Further increases in temperature, above $350^{\circ} \mathrm{F}$, changes the mode shape again to five circumferential waves and one half axial wave. The thermal variation has less effect on the changing mode shape since that part of the resultant load term is multiplied by a sine factor. Values for the variation which do alter the buckling are so high that materials with reasonable thermal conductivity would not maintain the given thermal state, and are not experienced in practical applications.

The mode shape into which the cylinder buckles is dependent not only on loading conditions but also on the cylinder geometry. To illustrate this effect, the baseline thermally-loaded configuration described above was altered geometrically. Consideration was first given to the ratio between the length and the radius of the cylinder. It was found that scaling this ratio did not result in comparable buckling loads or modes. For the same thickness, radially larger cylinders demonstrated less rigidity and the critical torsional loads were reduced while the number of waves in the mode shape increased.

A visual representation of the buckled shape of a cylinder under torsional load is shown in Fig. 2. The illustration is from the computational program Maple, based on the boundary condition shape function

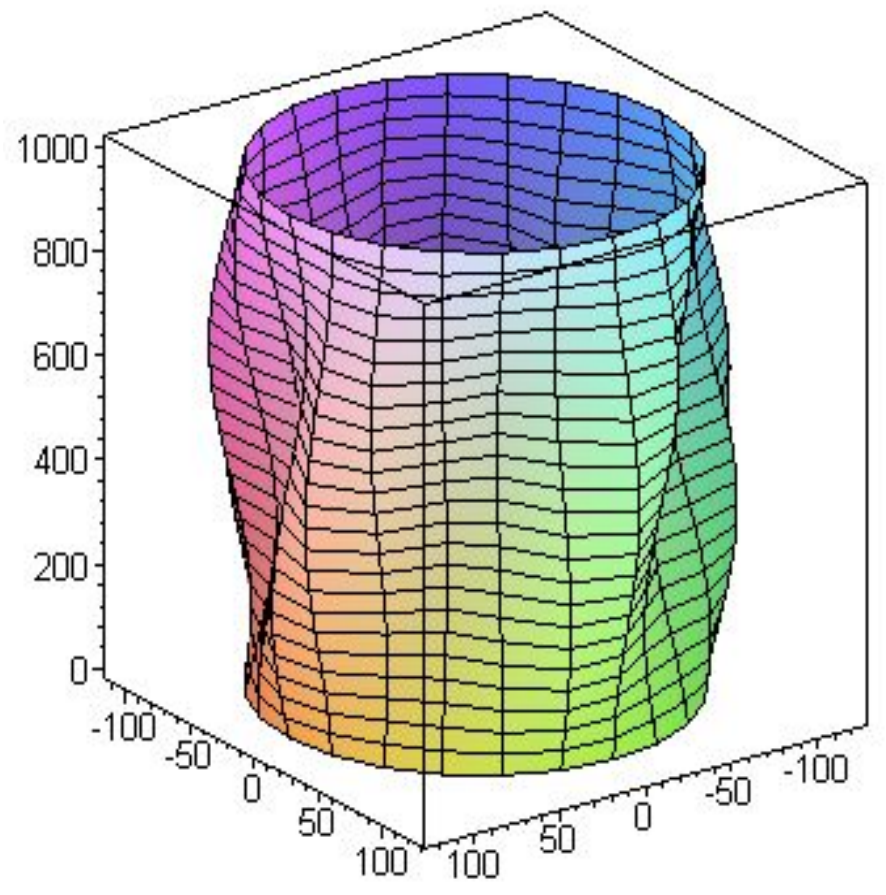

Figure 2: Thin-walled cylinder deformation under torsional load with clamped boundary conditions used in the Galerkin approximation, Eq. (21). For clarity, the mode shape with five full sinusoidal circumferential waves and one half sinusoidal axial wave has been chosen and the displacements have been exaggerated.

\section{Conclusion}

The analysis performed on the torsional stability of a thin-walled cylinder shows that, although a thermal load distribution does alter the torsional buckling load, the thermal contribution to the critical load is marginal. Thermal loading increases the strain energy and therefore reduces the critical torsional buckling load. Whereas the direct influence is slight, awareness of the role of the thermal strains in torsional buckling is important for understanding the behavior of cylindrical shells subjected to multiple-loading situations.

A mathematical model that accurately predicts the buckling behavior of thin-walled cylindrical shells under combined thermal (circumferential temperature distribution) and torsional loading has been presented. Further development of this model will incorporate additional loading states, such as due to forces generated on account of cylinder rotation.

\section{Acknowledgments}

The authors would like to thank Dr. John A. Charles for describing this problem and for his assistance with editing the manuscript.

\section{References}

${ }^{1}$ Lennon, R. F., and Das, P. K., “Torsional buckling behaviour of stiffened cylinders under combined loading,” Thin-Walled Structures, Vol. 38, No. 3, 2000, pp. 229, 245.

${ }^{2}$ Brush, D. O., and Almroth, B. O., Buckling of Bars, Plates, and Shells, McGraw-Hill, Inc., New York, 1975, Chaps. 5, 6.

${ }^{3}$ Donnell, L. H., “Stability of Thin-walled Tubes under Torsion,” NACA Rep. 479, 1933. 
${ }^{4}$ Batdorf, S. B., “A Simplified Method of Elastic Stability Analysis for Thin Cylindrical Shells,” NACA Rep. 874, 1947.

${ }^{5}$ Batdorf, S. B., Stein, M., and Schildcrout, M., "Critical Stress of Thin-Walled Cylinders in Torsion,” NACA TN 1344, 1947.

${ }^{6}$ Charles, J. A., “An Investigation of the Yankee Dryer “Out of Round” Condition,” Proceedings of the Tissue World Conference, Nice, France, March 2005 (submitted for presentation).

${ }^{7}$ Charles, J. A., "Precision Infrared Imaging for Yankee Dryers," Proceedings of the Tissue World Conference, Nice, France, March 2001. 